Manuskript „Bildgebende Verfahren” für die Zeitschrift Der MKG-Chirurg 1/2011

\title{
DOI 10.1007/s12285-010-0195-x
}

Korrespondenzadresse: Prof. Dr. P. Cattin

Medical Image Analysis Center (MIAC), Universität Basel, Universitätsspital Basel

Spitalstr. 21, 4031 Basel, Switzerland

philippe.cattin@unibas.ch

Weitere Autoren:

G. Schulz · M. Reyes

$\checkmark$ Bitte kreuzen Sie die gewünschten Optionen an und senden Sie dieses Formular an die oben angegebene E-Mail-Adresse oder Faxnummer. Dies ist auch dann wichtig, wenn Sie keine Korrekturen haben. Vielen Dank. Die Erteilung der Rechtseinräumung mit Ihrer Unterschrift ist für eine Veröffentlichung erforderlich!

\section{Imprimatur}

Bitte beachten Sie, dass nach Imprimatur keine weiteren Textkorrekturen, Änderungen der Autorenschaft oder der

Autorenreihenfolge möglich sind.

$\square \quad$ Hiermit gebe ich mein Manuskript direkt frei. Korrekturen sind nicht notwendig.

Ich habe einzelne Seiten des Manuskriptes korrigiert und diese der Antwort beigefügt. Nach ausgeführter Korrektur ist das Manuskript imprimiert.

Folgende Manuskript-Seiten habe ich beigefügt:

$\square \quad$ Diese Rückantwort enthält das komplette Manuskript mit entsprechenden Korrekturwünschen. Nach ausgeführter Korrektur ist das Manuskript imprimiert. Mir ist bewusst, dass größere Korrekturen oder Änderungen an der Textlänge ein späteres Publikationsdatum nach sich ziehen können.

\section{Sonderdrucke}

Ich wünsche kostenpflichtige Sonderdrucke meines veröffentlichten Manuskriptes. Das ausgefülle Formular „Sonderdruckbestellung“ liegt bei.

\section{Interessenkonflikt}

Die Angabe eines Interessenkonflikts ist seriöse Publikationspraxis. Bitte geben Sie alle finanziellen oder persönlichen Beziehungen von Ihnen und Ihren Co-Autoren zu Dritten an, deren Interessen vom Beitragsinhalt positiv oder negativ betroffen sein können, auch wenn aus Ihrer Sicht keine Beeinflussung stattfindet. Beispiele: finanzielle Verbindung zu Pharmafirmen, Beschäftigungsverhältnisse, Beratungs- und Referententätigkeiten, Honorare, Reisekostenübernahmen, Drittmittel, Aktienbesitz. Die Erklärung bezieht sich auf die Gegenwart und die vergangenen 5 Jahre und wird veröffentlicht.

Auch wenn kein Interessenkonflikt besteht, ist dies anzugeben. Sollte der Interessenkonflikt bereits im Manuskript genannt worden sein, erübrigt sich die Angabe an dieser Stelle.

$\square \quad$ Der korrespondierende Autor gibt an, dass kein Interessenkonflikt besteht.

Der korrespondierende Autor weist auf folgende Beziehung/en hin:

\section{Rechtseinräumung}

In Erweiterung des $§ 38$ Abs. 1 UrhG räume ich als erstgenannter Verfasser der Springer Science+Business Media Gruppe das ausschließliche, geografisch und zeitlich unbeschränkte Recht der Speicherung, Vervielfältigung, Verbreitung und Wiedergabe meines Beitrages (inkl. Bilder, Video- und Audiodateien) ein. Dies schließt das Recht zur Übersetzung und Bearbeitung ein und gilt für die Dauer des gesetzlichen Urheberrechts für alle Auflagen und Publikationen in gedruckter und elektronischer Form (z.B. online, offline, mobile Nutzung, embedded content). Springer ist berechtigt, die Nutzungsrechte am gesamten Beitrag und Teilen daraus in gedruckter und elektronischer Form wahrzunehmen und weiterzugeben.

Hiermit versichere ich - auch im Namen der Miturheber -, dass ich berechtigt bin, über die urheberrechtlichen Nutzungsrechte an dem o.g. Beitrag zu verfügen. Das Einverständnis abgebildeter Personen bzw. deren Erziehungsberechtigten liegt vor. 


\section{Sonderdruckbestellung}

Wenn Sie Sonderdrucke Ihres Artikels bestellen möchten, füllen Sie bitte dieses Formular aus und senden es zusammen mit Ihren Korrekturen an uns zurück. Sollten Sie erst nach dem Druck eines Heftes Sonderdrucke bestellen, beträgt die Mindestabnahme 300 Exemplare, da der Nachdruck erhöhten Aufwand bedeutet. Bitte beachten Sie, dass alle Anzeigen, die in der gedruckten Version Ihres Beitrags im Heft veröffentlicht werden, auch im Sonderdruck erscheinen.

Achtung: Ihre Bestellung kann nur bearbeitet werden, wenn eine Kreditkartennummer und Unterschrift vorliegen.

Hiermit bestelle ich:

\begin{tabular}{|l:l|}
\hline$\square$ 50 Exemplare & 250 EUR \\
\hline$\square$ 100 Exemplare & 300 EUR \\
\hline$\square 200$ Exemplare & 400 EUR \\
\hline$\square 300$ Exemplare & 500 EUR \\
\hline$\square 400$ Exemplare & 610 EUR \\
\hline$\square 500$ Exemplare & 720 EUR \\
\hline $\begin{array}{l}\square \text { über 500 } \\
\text { Exemplare }\end{array}$ & Wir erstellen Ihnen ein \\
individuelles Angebot.
\end{tabular}

(Preise inkl. Porto und Verpackung)

Bitte geben Sie Ihre VAT-Nummer an:

Kunden aus EU-Ländern ohne VAT-Nummer müssen den allgemein gültigen Steuerbetrag addieren

Bitte geben Sie Ihre Kreditkartennummer an:

$\square$ Eurocard/Access/Mastercard

$\square \quad$ American Express

$\square \quad$ Visa/Barclaycard/BankAmericard

Kartennummer (inkl. Prüfziffern):

$-----------------$

Gültig bis: _- $I_{-}$

Datum / Unterschrift:

\section{Rechnungsanschrift:}

$\square \quad$ siehe Lieferanschrift

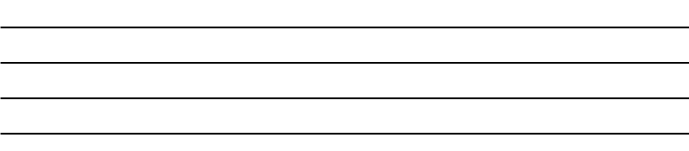

Für Autoren wohnhaft in Deutschland ist die Zahlung per Bankeinzug möglich:

Hiermit erteile ich Springer die

Abbuchungsgenehmigung von meinem Bankkonto über den Rechnungsbetrag bei Fälligkeit.

Kontonr.:

Bankleitzahl:

Bank:

Datum / Unterschrift:

Bitte verwenden Sie bei der Rechnungsstellung folgende Bestellnummer/folgendes Zeichen:

Bitte überprüfen Sie die oben angegebenen Daten und korrigieren Sie sie gegebenfalls. 


\begin{tabular}{|l|l|}
\hline DOI & $10.1007 / \mathrm{s} 12285-010-0195-x$ \\
\hline Copyright & Springer-Verlag -2011 \\
\hline
\end{tabular}

\section{Leitthema}

\section{Bildgebende Verfahren}

\section{Neue Methoden verändern die Chirurgie}

P. Cattin ${ }^{1} \cdot$ G. Schulz ${ }^{2} \cdot$ M. Reyes ${ }^{3}$

${ }^{1}$ Medical Image Analysis Center (MIAC), Universität Basel, Universitätsspital Basel, Basel, Switzerland

${ }^{2}$ Biomaterials Science Center (BMC), Universität Basel, Basel, Switzerland

${ }^{3}$ Institute for Surgical Technology and Biomechanics, Universität Bern, Bern, Switzerland

\section{Korrespondenzadresse}

Prof. Dr. P. Cattin

Medical Image Analysis Center (MIAC), Universität Basel, Universitätsspital Basel

Spitalstr. 21

4031 Basel

Switzerland

philippe.cattin@unibas.ch

\section{Prof. Dr. P. Cattin}

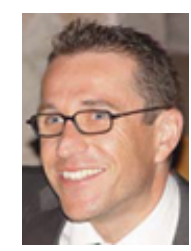

Online publiziert: Onlinedatum erscheint nach Freigabe

\section{Zusammenfassung}

Bildgebende Verfahren sind von enormer Bedeutung für die Mund-, Kiefer- und

Gesichtschirurgie. In diesem Artikel werden zwei bildgebende Verfahren genauer vorgestellt, 
von denen entscheidende Impulse im MKG-Bereich erwartet werden. Dies ist zum einen die multispektrale Computertomographie, die mittelfristig die Weichteilkontraste im CT verbessern wird. Längerfristig wird dann auch die Phasenkontrastbildgebung in konventionellen CT-Systemen zum Einsatz kommen. Da der Phasenkontrast nicht auf der Absorption der Röntgenstrahlen, sondern auf deren Ablenkung basiert, wird der Kontrast insbesondere im Weichgewebe sogar besser sein als bei der Magnetresonanzbildgebung.

\section{Schlüsselwörter}

Bildgebende Verfahren · Computertomographie $\cdot$ Multispektrale CT · Dual-Energy-CT ·

Differenzieller Phasenkontrast

\section{Imaging procedures}

\section{New methods modify surgery}

\section{Abstract}

Imaging methods play a crucial role in the field of craniomaxillofacial (CMF) surgery. This article describes two X-ray imaging modalities which will influence the field of CMF. In particular, the article introduces multi-spectral computed tomography (CT) which will bring better soft-tissue contrast in computed tomography. Although still in its early stages of development, differential phase-contrast imaging will be integrated into computed tomography systems in the near future. As phase-contrast imaging is independent of X-ray absorption and measures the real part of the refractive index of tissues, it offers even better soft tissue contrast than magnetic resonance imaging (MRI) microscopy.

\section{Keywords}

Imaging $\cdot$ Computed tomography $\cdot$ Multi-spectral CT $\cdot$ Dual-energy CT $\cdot$ Differential phase contrast

Die Mund-, Kiefer- und Gesichtschirurgie als Bereich der Spitzenmedizin ist auf hochaufgelöste und kontrastreiche dreidimensionale Bilder angewiesen. Die MKG-Chirurgie spielt aber nicht 


\section{Anforderungen an die zukünftige Bildgebung}

Die Mund-, Kiefer- und Gesichtschirurgie ist ein operatives Fach mit sehr hohen Anforderungen an das funktionale und ästhetische Resultat eines Eingriffs. Um diesen Anforderungen gerecht zu werden, wurden spezifische Werkzeuge in der computerunterstützten Chirurgie entwickelt und im klinischen Ablauf integriert. Parallel dazu sind die Ansprüche an die bildgebenden Verfahren ständig gestiegen. Neben Aufnahmen mit höherer Auflösung werden auch bessere Kontrast- und Rauschverhältnisse erwartet. Zweidimensionale Radiographien, wie sie jahrzehntelang verwendet wurden, reichen nicht mehr aus, um die neuen Möglichkeiten der Operationsplanung und Navigation zu nutzen. Zunehmend werden dreidimensionale CT-Daten für die Kephalometrie eingesetzt [1] . Multimodale Bildgebung und 3-D-Aufnahmeverfahren helfen dem Chirurgen, komplizierte Eingriffe an soliden Rapid-Manufacturing-Modellen, aber vermehrt auch mit rein virtuellen Werkzeugen zu planen [2]].

Die bei der Planung gewonnen Informationen werden dann in Verbindung mit optischen Tracking-Technologien und Patientenregistrierungsverfahren zur intraoperativen Navigation verwendet. Diese Verfahren ermöglichen bereits heute eine Genauigkeit von $<1 \mathrm{~mm}[\underline{3}]$.

\section{$>$ Diese Verfahren ermöglichen bereits heute eine Genauigkeit von $<1 \mathrm{~mm}$}

Neben der reinen Eingriffsplanung werden in Zukunft virtuelle Simulationswerkzeuge zur Routinevorbereitung jedes größeren chirurgischen Eingriffs gehören. Noch sind aber die dazu notwendigen bildgebenden Verfahren mit der anschließenden Nachbearbeitung - wie z. B. der Segmentierung - nicht weit genug entwickelt. Der Aufwand und die daraus resultierenden Kosten sind noch zu hoch.

In der Forschung wird aber bereits an den zukünftigen Planungs- und Simulationswerkzeugen gearbeitet: in der Schweiz beispielsweise im Nationalen Forschungsschwerpunkt NCCR CO-ME (http://co-me.ch) an verbesserten, präziseren Verfahren zur patientenspezifischen automatischen Segmentierung der Knochen und Weichteile sowie an Neuerungen der intraoperativen Echtzeitnavigation. 
In Abb. 1 wird der Vergleich der Simulation einer Umstellungsosteotomie mit dem realen postoperativen Resultat veranschaulicht [4]. Das Maß der Abweichung zwischen Simulation und postoperativem Resultat lässt sich mithilfe der Farbkodierung präzise darstellen.

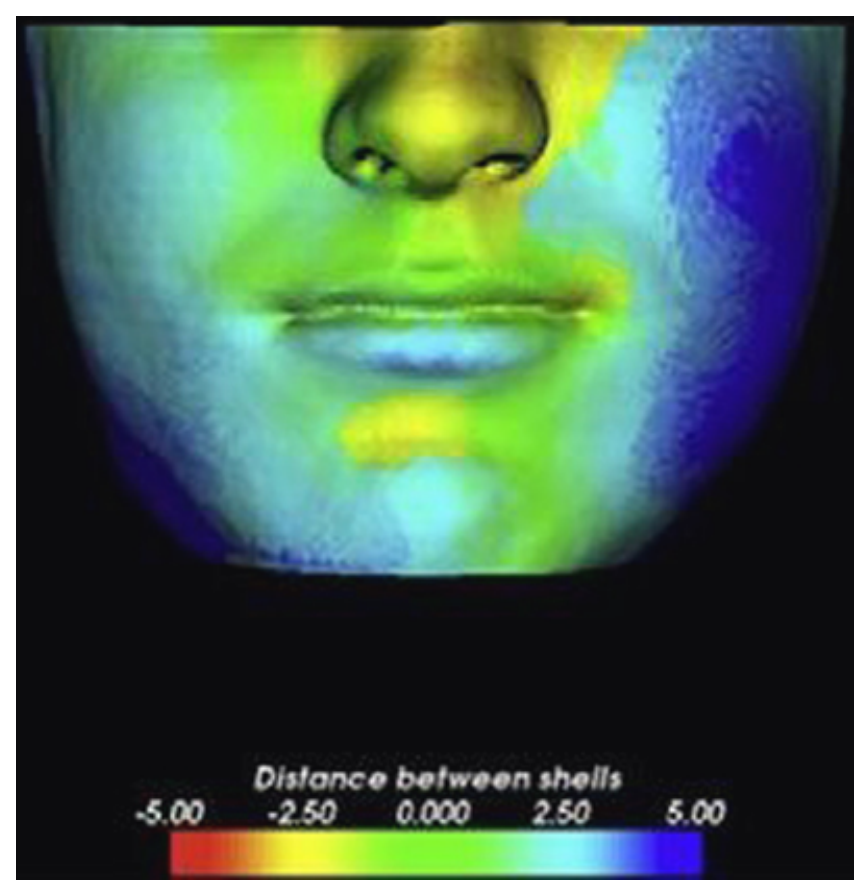

Abb. 1 Farbkodierte Distanzkarte von einer simulierten Umstellungsosteotomie im Vergleich zum Resultat des chirurgischen Eingriffs. (Aus [4])

Nicht nur die Chirurgen, auch die Patienten haben ein Interesse an verbesserten bildgebenden Verfahren, etwa in der Frage einer geringeren Strahlenbelastung beim CT. Zur Reduktion der Röntgendosis geeignet sind beispielsweise lokale Verfahren, wie die digitale Volumentomographie (DVT), auch als ,cone beam CT“ bezeichnet. Die geringere Strahlendosis wird mit einem kleineren Aufnahmevolumen, nichtstandardisierten Graustufen im Bild (keine Hounsfield-Einheiten), einem geringeren Weichteilkontrast sowie einem schlechteren Signal-zu-Rausch-Verhältnis erkauft. Für einige Anwendungen im MKG-Bereich sind diese Aufnahmen ausreichend.

\section{! Für die Segmentierung und Simulationen genügt die DVT jedoch nicht.}

Die weitere Entwicklung und Verfeinerung der patientenspezifischen Planungs-, Simulationsund Navigationswerkzeuge hängt von den Fortschritten anderer Verfahren ab. Mittelfristig dürfte sich die relativ junge Technik der multispektralen CT durchsetzen. Die Aufnahme von verschiedenen spektralen Kanälen erlaubt eine bessere Unterscheidung der einzelnen Gewebetypen dank des verbesserten Weichteilkontrasts. 
Längerfristig verspricht die Phasenkontrastbildgebung interessante Möglichkeiten: Mit ihr lässt sich die Röntgendosis weiter reduzieren, ohne dass dabei das Signal-RauschVerhältnis negativ beeinflusst wird. Die Phasenkontrastbildgebung erlaubt auch die genauere Unterscheidung von verschiedenen Weichgeweben und vereinfacht so die künftige Operationsplanung.

\section{Hintergrund}

Sowohl in der konventionellen planaren Röntgendiagnostik als auch in der

Computertomographie wird der Bildkontrast durch die lokale Röntgenabsorption in den verschiedenen Gewebeschichten erzeugt. Die Absorption hängt zwar von dem verwendeten Röntgenspektrum ab, der Unterschied bei verschiedenen Weichgeweben ist aber in der Regel sehr klein. Daraus resultiert der bekanntermaßen schlechte Kontrast in den Weichgeweben. Für die patientenspezifische Operationsplanung werden aber neben einer guten räumlichen Auflösung auch gute Kontrastverhältnisse vorausgesetzt.

\section{$>$ Für die Operationsplanung werden gute Kontrastverhältnisse vorausgesetzt}

Mehrere Verfahren zur Kontrastverbesserung wurden bereits vorgeschlagen bzw. werden derzeit aktiv erforscht. Für den medizinischen Bereich sind vor allem zwei CT-basierte Verfahren von besonderem Interesse: die multispektrale CT-Bildgebung sowie die Phasenkontrastbildgebung.

\section{Multispektrales CT}

Um den schwachen Weichgewebekontrast zu verbessern, wird in den Dual-Energy-CT(DECT)-Systemen die Absorption bei unterschiedlichen Wellenlängen der Röntgenstrahlung gemessen. Dieses Prinzip basiert auf Ideen, die in den 1980er Jahren für die QCTKnochendichtemessung entwickelt wurden.

In Abb. 2 werden vier verschiedene Spektren von Röntgenröhren dargestellt, wie sie typischerweise bei Beschleunigungsspannungen von $80 \mathrm{kV}, 100 \mathrm{kV}, 120 \mathrm{kV}$ und $140 \mathrm{kV}$ erhalten werden. Das Spektrum setzt sich aus der Bremsstrahlung und der charakteristischen Röntgenstrahlung zusammen. Letztere ist vom Target-Material abhängig. Die Spektren wurden zusätzlich mit einem Aluminiumfilter im Strahlengang ,aufgehärtet“, genauer, die 
schwach energetischen Photonen wurden bevorzugt gefiltert, da sie nur die Röntgendosis für den Patienten erhöhen, aber kaum Einfluss auf die Bildgebung haben.

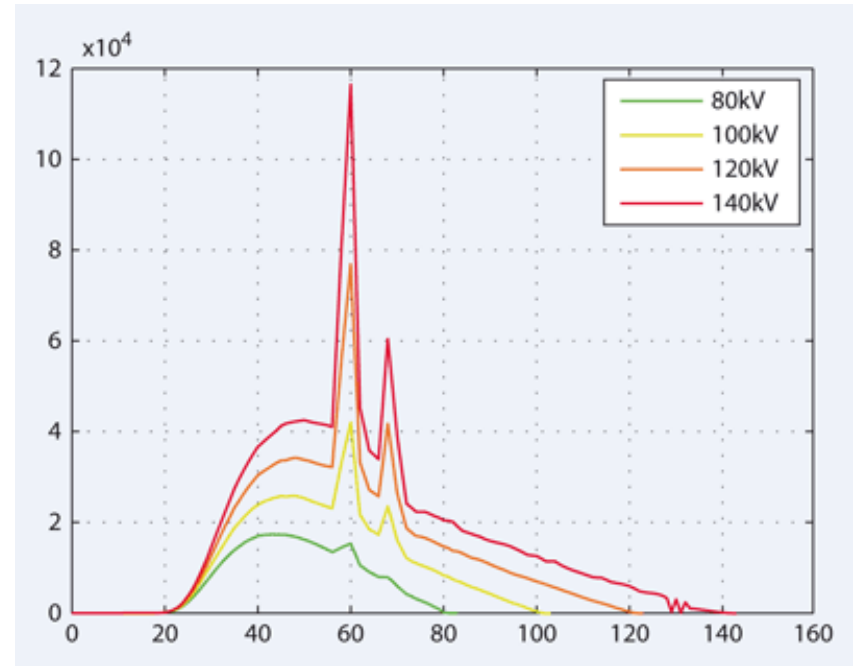

Abb. 2 Simulierte Röntgenspektren bei unterschiedlichen Beschleunigungsspannungen

Misst man mit zwei dieser Spektren die lokale Röntgenabsorption von verschiedenen Geweben, so erhält man einen besseren Kontrast (Abb. 3). Kontrastmittel in Blutgefäßen lassen sich so beispielsweise von Kalkablagerungen unterscheiden.

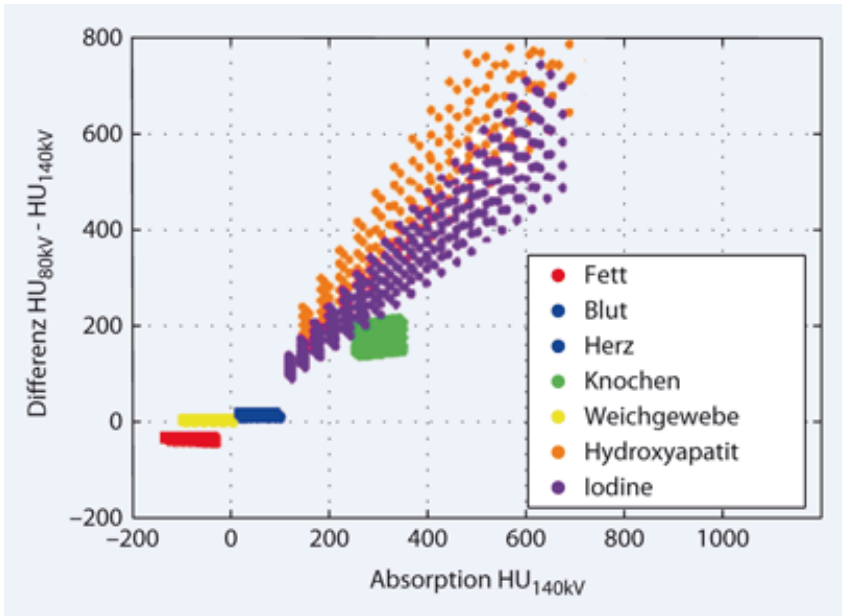

Abb. 3 Wie diese Simulation zeigt, erlaubt die Messung der Röntgenabsorption mit unterschiedlichen Energien eine bessere Unterscheidung der verschiedenen Gewebearten

Mit den kürzlich eingeführten DECT-Systemen strebt man genau diese Verbesserung des Bildkontrasts an.

\section{! Entscheidend dabei ist die „quasi“ gleichzeitige Aufnahme der Absorption bei unterschiedlichen Spektren.}

So können die Bewegungsartefakte minimiert werden. 
Alle namhaften Hersteller haben bereits eine Lösung für DECT-Systeme entwickelt. Diese Entwicklungen basieren auf unterschiedlichen Ansätzen. Die Fa. Siemens hat im Jahre 2005 als erste ein 64-Zeilen-CT (Somatom Definition) eingeführt, das über zwei unabhängige Röntgenröhren und Detektorarrays in derselben Gantry verfügt (Dual-Source-CT, DSCT, Abb. 4a). Durch die zwei um $90^{\circ}$ versetzt angeordneten Röntgenröhren kann auf der einen Seite die Aufzeichnungszeit für ein klassisches CT halbiert werden, sofern die beiden Röhren mit der gleichen Spannung betrieben werden. Bei unterschiedlichen Röhrenspannungen ermöglicht das System die Aufnahme der Absorption, bei zwei verschiedenen Röhrenspannungen in nur einem Durchgang, also „quasi“ zeitgleich.

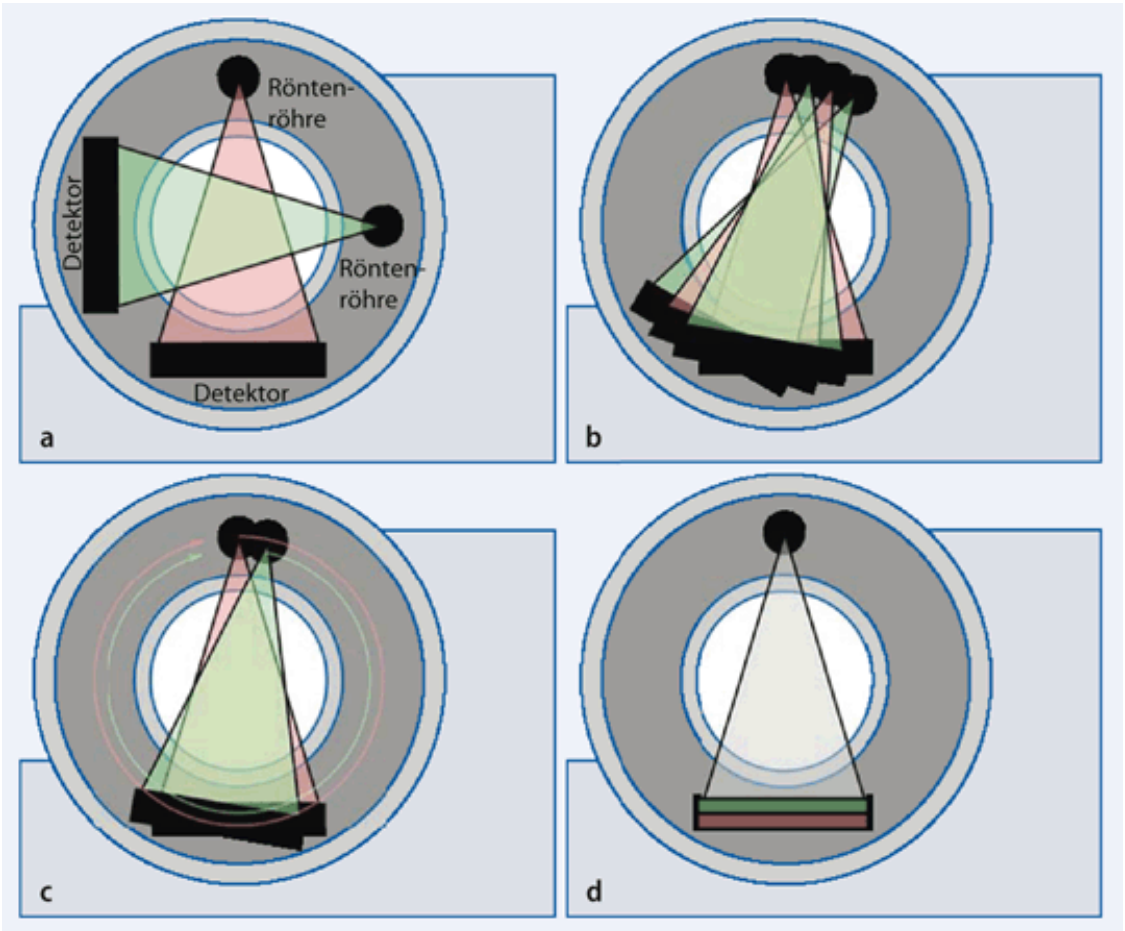

Abb. 4 a Siemens hat zwei Quellen integriert. b General Electric schaltet in kurzen Abständen die Röhrenspannung um. c Toshiba schaltet die Röhrenspannung nach jeder Umdrehung um. d Philips verwendet einen Detektor mit mehreren Layern, die auf unterschiedliche Energien empfindlich sind

Einen etwas anderen Ansatz verfolgt die Fa. General Electric, die für ihre MultienergieAufnahmetechnik (CT750 HD) nur mit einer einzigen Röntgenröhre und einem Detektor arbeitet. Die Röhrenspannung wird periodisch im Millisekundentakt zwischen den zwei gewählten Energien hin- und hergeschaltet (Abb. 4b). Diese Lösung ist zwar mechanisch einfacher, stellt aber wesentlich höhere Anforderungen an die Elektronik und die Röntgenröhrentechnik. 
Der Ansatz von Toshiba ist ähnlich, nur werden die Beschleunigungsspannungen nach jeder vollen Rotation gewechselt (Abb. 4c). Dieser Ansatz ist technisch am einfachsten zu lösen, erfordert aber bessere Bewegungskompensation, da die Zeit zwischen der Aufnahme mit den beiden Röntgenspektren relativ lange ist.

Das multispektrale CT (MSCT) von Philips verwendet ebenfalls nur eine Röntgenröhre mit konstanter Röhrenspannung. Im Unterschied zu den anderen Herstellern ist der Detektor von Philips mehrschichtig aufgebaut, wobei die oberste Schicht den Anteil an tief energetischen Photonen misst und die darunterliegenden Schichten den Anteil an höher energetischen Photonen erfassen (Abb. 4d). Im klinischen Einsatz sind vorerst nur Detektoren, die zwei Energieniveaus messen, doch es existieren schon Prototypen mit Mehrschichtdetektoren.

\section{$>$ Ziel der MSCT-Technik ist eine detailliertere Analyse des Gewebes}

Obwohl die Idee und die theoretischen Grundlagen des MSCT schon seit Längerem bekannt sind, haben erst die Entwicklungen der letzten Jahre einen klinischen Vorteil gebracht. Die Anzahl der klinischen Anwendungen der MSCT-Technik wächst stetig. Wir befinden uns erst am Anfang dieser Entwicklung. Für eine abschließende Bewertung des vollen Potenzials der DECT-Technologie ist es noch zu früh. Schlussendliches Ziel ist es aber, anhand der spektralen Aufnahmen das Gewebe in seiner Dichte und chemischen Zusammensetzung detaillierter zu analysieren.

Ein Problem ist noch die im Vergleich zur konventionellen CT-Afnahme je nach Hersteller mehr oder weniger erhöhte Röntgendosis. Hier muss die für den Patienten geringste Röntgendosis gefunden werden, ohne auf Kontrast oder Ortsauflösung zu verzichten.

\section{Phasenkontrast-CT}

Im Unterschied zu der konventionellen Röntgenbildgebung wird bei den Phasenkontrastverfahren nicht die Absorption der Röntgenstrahlen, sondern die Elektronendichteverteilung durch die Ablenkung der Röntgenstrahlen an der Materie gemessen. Phasenkontrastmessungen sind deutlich aufwendiger und erreichen nicht ganz die Ortsauflösung der Absorptionsmessungen. Sie liefern aber einen deutlich besseren Kontrast, was insbesondere für geringe Dichteunterschiede wie im Gehirn wichtig ist. Man spricht hier auch vom Vorteilsfaktor, der insbesondere für Materialien mit kleiner Ordnungszahl in der 
Größenordnung $10^{3}$ liegt [5]. Bereits 1965 wurden mit einem Röntgeninterferometer erste Phasenkontrastradiographien gezeigt [6] .

Während der Absorptionskontrast etwa mit der 4. Potenz der Ordnungszahl des zu untersuchenden Objekts zunimmt, wächst der Phasenkontrast nur linear mit der Ordnungszahl [7]. Das führt dazu, dass man Weichgewebe in der Umgebung von Knochen leichter sichtbar machen kann. Die ersten Röntgentomogramme im Phasenkontrastmodus wurden interferometrisch gemessen []ㅡ. Unterschiede im Phasenkontrast der leichten Elemente sind häufig durch Dichtevariationen gegeben. Wasserstoffhaltige Verbindungen erzeugen einen besonders starken Phasenkontrast. Diese Eigenschaft des Wasserstoffs, die auf dem großen Masse-zu-Ladung-Verhältnis beruht, ist bereits von der NMR-Tomographie her bekannt.

\section{! Deshalb ist der Phasenkontrast besonders geeignet, Strukturen innerhalb der Weichgewebe zu detektieren.}

Inzwischen gibt es verschiedene Ansätze für Phasenkontrastmessungen. Jüngst wurde eine neue Methode etabliert - die sog. gitterbasierte Interferometrie [9]. An Synchrotronquellen wurden verschiedene menschliche Weichgewebe (Thalamus [11], Harnröhren [12]) erfolgreich untersucht, die sogar die Visualisierung der Purkinje-Zellen im Kleinhirn ohne jeglichen Einsatz von Kontrastmitteln ermöglichte [13]. Diese auf Synchrotronstrahlung basierenden microCT $(\mathrm{SR} \mu \mathrm{CT})$ sind aber für den Einsatz am lebenden Gewebe nicht geeignet, weil für die Mikrometerauflösung eine hohe Photonendichte notwendig ist. Bisher ist die Probengröße auf einige wenige Zentimeter Durchmesser beschränkt.

Verzichtet man auf die hohe Ortsauflösung, kann man diese Methode aber durchaus für In-vivo-Untersuchungen einsetzen. Dank eines Wissenschaftlerteams vom Paul Scherrer Institut (PSI) in Villigen, Schweiz, lässt sich dieser Ansatz auch mit konventionellen Mitteln durch Einsatz eines zusätzlichen Gitters anwenden [14]. Das PSI-Team konnte zeigen, dass man Phasenkontrast nicht nur mit dem aufwendig zu erzeugenden monochromatischen Licht des Synchrotrons, sondern auch mit der herkömmlichen Bremsstrahlung erzeugt, durch eine konventionelle Röntgenröhre messen kann. Das bedeutet, durch den Einbau von drei Gittern kann mit denselben Röntgenröhren, wie sie beispielsweise im MSCT zum Einsatz kommen, gearbeitet werden.

> Auch konventionelle Röntgenröhren können

Phasenkontrastbilder erzeugen 
Das Team vom PSI konnte dabei erstmals demonstrieren, wie man mit Röntgenröhren, trotz kurzer Koheränzlänge, effizient quantitative Phasenkontrastbilder erhalten kann. Dies lässt sich mithilfe von drei Gittern im Strahlengang wie beim „Talbot grating interferometer“ 5) erreichen. Das erste Gitter G0 trennt dabei die - einige Millimeter große - Röntgenquelle in viele Linienquellen auf. Diese sind zwar unter sich inkoheränt, aber in sich haben sie eine höhere Koheränz. Das zweite Gitter G1 ist so gebaut, dass es eine Phasenverschiebung von $\pi$ auf der einkommenden Strahlung bewirkt. Diese Phasenverschiebung erzeugt ein Interferenzmuster hinter dem Gitter G1. Änderungen im Brechungsindex in Teilen der Probe bewirken nun laterale Verschiebungen in diesem Interferenzmuster. Durch Messen dieser Verschiebungen lässt sich der differenzielle Phasenkontrast bestimmen. Da die Auflösung konventioneller Röntgendetektoren aber bei Weitem nicht ausreicht, um das Interferenzmuster korrekt wiederzugeben, wird noch ein Analysengitter G2 benötigt. Typischerweise wird dann eines der beiden Gitter (G1 oder G2) über eine Periode des Interferenzmusters geschoben und dabei werden mindestens vier Aufnahmen gemacht. Die bei jedem Pixel beobachtete Intensitätsänderung wird dann verwendet, um den differenziellen Phasenkontrast zu berechnen. Mit leichten Modifikationen können schließlich die üblichen Rekonstruktionsalgorithmen verwendet werden. Der außerordentlich gute Kontrast für das menschliche Kleinhirn wird in Abb. 6 veranschaulicht. Hier werden virtuelle Schnitte für Absorptions- und Phasenkontrast mit dem Schnitt der hochaufgelösten MR-Mikroskopie verglichen.

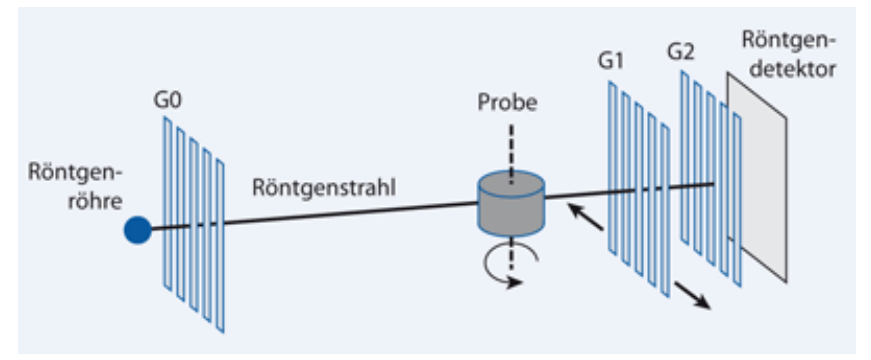

Abb. 5 Aufbau eines Talbot-Gitterinterferometers zur Phasenkontrastmessung 

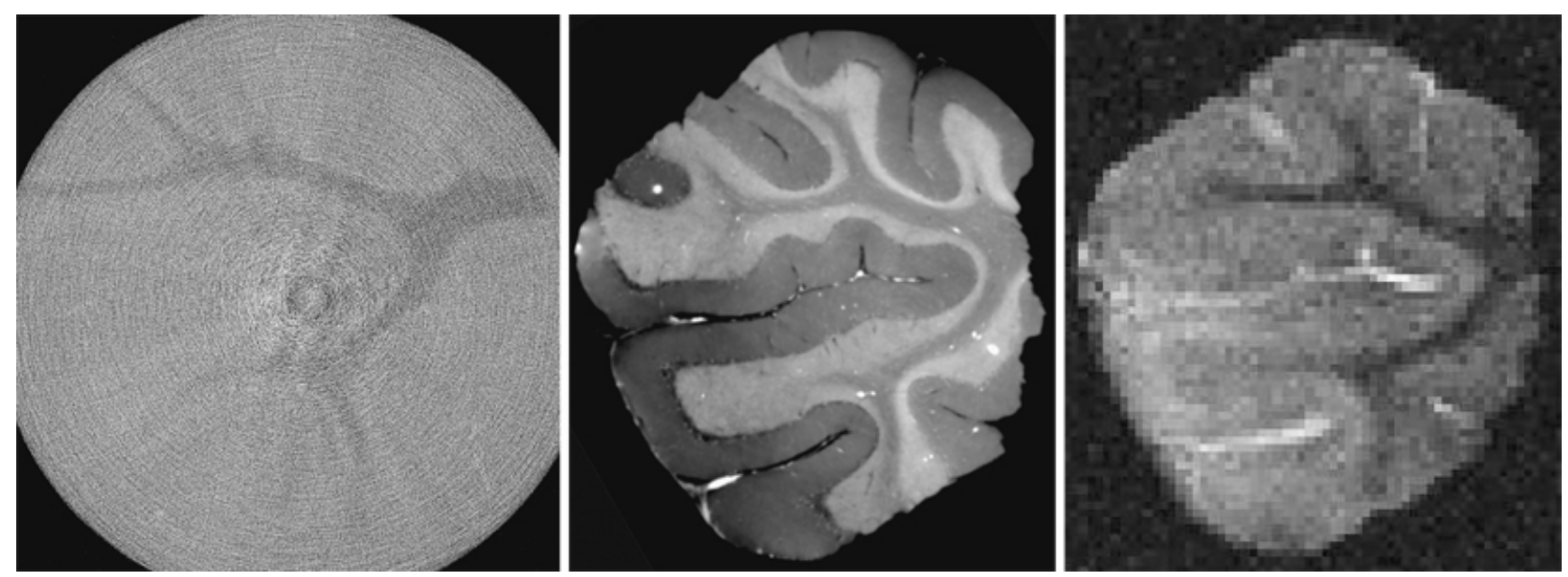

Abb. 6 Am Beispiel des menschlichen Kleinhirns. Virtueller Schnitt aus a

Absorptionskontrasttomogramm, b Phasenkontrasttomogramm, c MR-Mikroskopie

\section{Fazit}

- Dank neuer technologischer Entwicklungen ist die Vielfalt bildgebender Verfahren gestiegen. Die Herausforderung der Zukunft wird es sein, für jeden Patienten oder eine bestimmte Patientengruppe das jeweils beste bildgebende Verfahren anzuwenden.

- Das erfordert eine entsprechende Weiterbildung des medizinischen Personals und auch eine signifikante Reduktion der anfallenden Kosten.

- Neue Modalitäten werden sich nur durchzusetzen, wenn sie den bereits etablierten und bewährten Methoden deutlich überlegen sind.

- Nicht zu unterschätzen ist der Modernisierungsdruck durch die Patienten. Mit steigender Mobilität und besserer Information werden sie sich vermehrt für die Behandlungszentren mit dem ihres Erachtens besten und modernsten Behandlungskonzept entscheiden.

- Der MKG-Chirurgie stehen spannende Jahrzehnte bevor, in denen erfahrene Chirurgen neue bildgebende Verfahren zum Wohl der Patienten auswählen und einsetzen werden.

\section{Interessenkonflikt}

\section{Literatur}

1. Swennen GRJ, Schutyser F, Hausamen JE (2006) Three-dimensional cephalometry - a color atlas and manual. Springer, Berlin Heidelberg New York 
2. Krol Z, Chapuis J, Schwenzer-Zimmerer K et al (2005) Preoperative planning and intraoperative navigation in the reconstructive craniofacial surgery. J Med Inform Technol 9:83-89

3. Juergens P, Klug C, Ewers R, Schicho K et al (2011) Navigation guided harvesting of autologous iliac crest graft for mandibula reconstruction. J Oral Maxillofac Surg (in press)

4. Kim H, Jurgens P, Nolte LP, Reyes M (2010) Anatomically-driven soft-tissue simulation strategy for cranio-maxillofacial surgery using facial muscle template model. Med Image Comput Comput Assist Interv 6361:61-68

5. Bonse U (1997) Röntgen-Mikrotomographie. Phys Biol 53(3):211-214

6. Bonse U, Hart M (1965) An X-ray interferometer. Appl Phys Lett 6:155-156

7. Bonse U, Busch F (1996) X-ray computed microtomography ( $\mu \mathrm{CT}$ ) using synchrotron radiation (SR). Prog Biophys Mol Biol 65:133-169

8. Momose A (2003) Demonstration of X-ray Talbot interferometry. Jpn J Appl Phys 42:L866-L868

9. Weitkamp T, Diaz A, David C et al (2005) X-ray phase imaging with a grating interferometer. Opt Expr 13:6296-6304. DOI 10.1364/OPEX.13.006296

10. Weitkamp T, David C, Bunk O et al (2008) X-ray phase radiography and tomography of soft tissue using grating interferometry. Eur J Radiol 68S:13-17

11. Schulz G, Morel A, Imholz MS et al (2010) Evaluating the microstructure of human brain tissues using synchrotron radiation-based micro computed tomography. Proc SPIE 7804

12. Müller B, Schulz G, Herzen J et al (2010) Morphology of urethral tissues. Proc SPIE 7804

13. Schulz G, Weitkamp T, Zanette I et al (2010) High-resolution tomographic imaging of a human cerebellum: comparison of absorption and grating based phase contrast. J R Soc Interface 7(53):1665-1676

14. Pfeiffer F, Weitkamp T, Bunk O, David C (2006) Phase retrieval and differential phasecontrast imaging with low-brilliance X-ray sources. Nat Phys 2:258-261 